EGU2020-8583

https://doi.org/10.5194/egusphere-egu2020-8583

EGU General Assembly 2020

(c) Author(s) 2021. This work is distributed under

the Creative Commons Attribution 4.0 License.

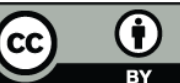

\title{
Integrated field-agent based modelling using the LUE scientific data base
}

Oliver Schmitz, Kor de Jong, and Derek Karssenberg

Utrecht University, Faculty of Geosciences, Physical Geography, Utrecht, Netherlands (o.schmitz@uu.nl)

The heterogeneous nature of environmental systems poses a challenge to researchers constructing environmental models. Many simulation models of integrated systems need to incorporate phenomena that are represented as spatially and temporally continuous fields as well as phenomena that are modelled as spatially and temporally bounded agents. Examples include moving animals (agents) interacting with vegetation (fields) or static water reservoirs (agents) as components of hydrological catchments (fields). However, phenomena bounded in space and time have particular properties mainly because they require representation of multiple (sometimes mobile) objects that each exist in a small subdomain of the space-time domain of interest. Moreover, these subdomains of objects may overlap in space and time such as interleaving branches due to tree crown growth. Efficient storage and access of different types of phenomena requires an approach that integrates representation of fields and objects in a single data model.

We develop the open-source LUE data model that explicitly stores and separates domain information, i.e. where phenomena exist in the space-time domain, and property information, i.e. what attribute value the phenomenon has at a particular space-time location, for a particular object. Notable functionalities are support for multiple spatio-temporal objects, time domains, objects linked to multiple space and time domains, and relations between objects. The design of LUE is based on the conceptual data model of de Bakker (2017) and implemented as a physical data model using HDF5 and C++ (de Jong, 2019). Our LUE data model is part of a new modelling language implemented in Python, allowing for operations accepting both fields and agents as arguments, and therefore resembling and extending the map algebra approach to field-agent modelling.

We present the conceptual and physical data models and illustrate the usage by implementing a spatial agent-based model simulating changes in human nutrition. We thereby consider the interaction between personal demand and supply of healthy food of nearby stores as well as the influence of agent's social network.

References:

de Bakker, M. P., de Jong, K., Schmitz, O., \& Karssenberg, D. (2017). Design and demonstration of a data model to integrate agent-based and field-based modelling. Environmental Modelling \& Software, 89, 172-189. https://doi.org/10.1016/j.envsoft.2016.11.016 
de Jong, K., \& Karssenberg, D. (2019). A physical data model for spatio-temporal objects.

Environmental Modelling \& Software. https://doi.org/10.1016/j.envsoft.2019.104553

LUE source code repository: https://github.com/pcraster/lue/ 